\title{
GMRES for the differentiation operator
}

\author{
Sheehan Olver \\ Oxford University Computing Laboratory, Wolfson Building, Parks Road, Oxford, UK \\ Sheehan.0lver@sjc.ox.ac.uk
}

\begin{abstract}
We investigate using the GMRES method with the differentiation operator. This operator is unbounded, and thus does not fall into the framework of existing Krylov subspace theory. We establish conditions under which a function can be approximated by its own derivatives in a domain of the complex plane. These conditions are used to determine when GMRES converges. This algorithm outperforms traditional quadrature schemes for a large class of highly oscillatory integrals, even when the kernel of oscillations is unknown.
\end{abstract}

Keywords GMRES, highly oscillatory integrals.

Oxford University Computing Laboratory

Numerical Analysis Group

Wolfson Building

Parks Road

Oxford, England OX1 3QD

May, 2008 


\section{Introduction}

The subject of this paper is finding an antiderivative of a smooth function $f$ in a set $\Omega$ of the complex plane; in other words we wish to find a particular solution $v$ to the following differential equation:

$$
\mathcal{D} v=f \quad \text { for } \quad f \in C^{\infty}[\Omega] .
$$

Note that we do not care which particular solution we find, as all solutions to this equation differ by a constant. Any smooth first order ordinary differential equation can be put into this form, since, for any function $a$,

$$
\mathcal{D} v=f \mathrm{e}^{a} \quad \Rightarrow \quad\left(\mathcal{D}+a^{\prime}\right) w=f \quad \text { for } \quad w=v \mathrm{e}^{-a} .
$$

We will employ GMres to find a solution to (1.1). Define the $n$th Krylov subspace as

$$
\mathcal{K}_{n}[f]=\operatorname{span}\left\{f, \mathcal{D} f, \mathcal{D}^{2} f, \ldots, \mathcal{D}^{n-1} f\right\}=\operatorname{span}\left\{f, f^{\prime}, f^{\prime \prime}, \ldots, f^{(n-1)}\right\}
$$

We then let $v_{n}^{\mathrm{G}}$ be an element in $\mathcal{K}_{n}[f]$ which minimizes the norm of the residual

$$
\left\|\mathcal{D} v_{n}^{\mathrm{G}}-f\right\|
$$

for some suitable norm $\|\cdot\|$. We employ the following notation for norms: we minimize with respect to the norm $\|\cdot\|$, which results from an inner product $\langle\cdot, \cdot\rangle$ defined over $\Omega$; while $\|\cdot\|_{\Omega}$ is the supremum norm over $\Omega$. The choice of inner product is discussed in Section 3. We will refer to this method as differential GMRES, to distinguish it from its finite dimensional and bounded operator counterparts.

The algorithm we use for computing $v_{n}^{\mathrm{G}}$ is virtually the same as the finite dimensional version of GMRES, with the inner product $\langle\cdot, \cdot\rangle$ used in place of the vector dot product. Thus we determine $v_{n}^{\mathrm{G}}$ as follows $[\mathbf{9}]$ :

(1) Using continuous Arnoldi iteration, determine an orthonormal basis $\boldsymbol{q}_{n}=\left(q_{1}, \ldots, q_{n}\right)$ and an $(n+1) \times n$ Hessenberg matrix $H_{n}$ such that

$$
\mathcal{D} \boldsymbol{q}_{n}=\boldsymbol{q}_{n+1} H_{n}
$$

(2) Compute coefficients $\boldsymbol{c}=\left(c_{1}, \ldots, c_{n}\right)^{\top}$ by solving the finite-dimensional least squares problem

$$
H_{n} c \approx\|f\| e_{1}
$$

(3) Define

$$
v_{n}^{\mathrm{G}}=\boldsymbol{q}_{n} \boldsymbol{c}=\sum_{k=1}^{n} c_{k} q_{k} .
$$


This does indeed compute an element with minimal norm:

$$
\left\|\mathcal{D} v_{n}^{\mathrm{G}}-f\right\|=\left\|\boldsymbol{q}_{n+1} H_{n}-\right\| f\left\|\boldsymbol{q}_{1}\right\|=\left\|\boldsymbol{q}_{n+1}\left[H_{n}-\|f\| \boldsymbol{e}_{1}\right]\right\|=\left\|H_{n}-\right\| f\left\|\boldsymbol{e}_{1}\right\|
$$

Once $v_{n}^{\mathrm{G}}$ is computed, we can compute definite integrals over an interval $(a, b)$ using

$$
Q_{n}^{\mathrm{G}}=v_{n}^{\mathrm{G}}(b)-v_{n}^{\mathrm{G}}(a) .
$$

A great deal of research has been carried out on Krylov subspace methods for bounded operators [5]. But differential operators are unbounded, and the existing theory on convergence fails. Thus the question is: when does differential GMRES converge, or in other words, which smooth functions can be approximated by their own derivatives in $\Omega$ ? We denote the set of such functions by $\mathcal{G}[\Omega]$ :

Definition 1.1 $\mathcal{G}[\Omega]$ is the set of functions $f \in \mathbb{C}^{\infty}[\Omega]$ such that, for any $\epsilon>0$, there exists an $n$ and function $g \in \mathcal{K}_{n}[f]$ such that

$$
\left\|f-g^{\prime}\right\|_{\Omega}<\epsilon
$$

This set is not all of $C^{\infty}[\Omega]$ : nonzero polynomials are not in $\mathcal{G}[\Omega]$, nor are exponentials plus nonzero polynomials. We will establish in Section 2 several conditions which ensure that $f \in \mathcal{G}[\Omega]$. These conditions rely on the following set $\mathcal{P}[\Omega]$ :

Definition 1.2 For a sequence of balls $\boldsymbol{B}=\left\{B_{1}, B_{2}, \ldots\right\}$ in the complex plane, $\mathcal{P}[\Omega, \boldsymbol{B}]$ is the set of analytic functions $f$ such that $\|f\|_{\Omega+B_{k}} \rightarrow 0$ as $k \rightarrow \infty$.

Definition 1.3 $\mathcal{P}[\Omega]$ is the union of $\mathcal{P}[\Omega, \boldsymbol{B}]$ over all sequences $\boldsymbol{B}$.

In other words, $f \in \mathcal{P}[\Omega]$ if it decays in the complex plane at a sequence of domains larger than $\Omega$.

We will find that $f \in \mathcal{G}[\Omega]$ if $\Omega$ is bounded and any of the following conditions hold (here $D \supset \Omega$ denotes an open connected domain in which $f$ is analytic):

(1) $\quad f$ is entire and $f \in \mathcal{P}[\Omega]$;

(2) $f \in \mathcal{P}[\Omega, \boldsymbol{B}]$, where $\boldsymbol{B}$ and the point zero lie within an open connected domain $U$ such that $U+\Omega \subset D$;

(3) $\Omega$ is an interval, $f$ is analytic apart from a finite number of singularities and $f \in \mathcal{P}[\Omega]$.

(4) $\Omega$ is a real interval and $f$ is entire, has only polynomial growth on $\mathbb{R}$ and is not a polynomial plus a sum of nonzero exponentials with imaginary exponents [8].

When $\Omega$ is unbounded, we require the following condition to ensure that $f \in \mathcal{G}[\Omega]$ : 
- Condition (2) is satisfied and $\|f\|_{u+\Omega}<\infty$ for every $u \in U$.

A plethora of other schemes exist for solving (1.1) - trapezium rule, Simpson's rule, Gauss-Legendre quadrature, Gauss-Kronrod rules and so on-typically by computing the integral

$$
\int_{0}^{z} f(t) d t
$$

Many of these methods converge geometrically for analytic functions $f$. This raises the question: why do we need yet another method, which is nonlinear and does not even work for polynomials? There are two reasons we are interested in differential GMRES:

(1) It can be more accurate in the presence of oscillations, and in certain circumstances drastically so. Numerical results supporting this claim and an explanation why this is true are presented in Section 3;

(2) The use of Krylov subspace methods for differential equations is an area of research that has been neglected. Problem (1.1) seems to be a reasonable starting place for developing a theoretical framework.

There are certain cases when differential GMRES is not effective as a quadrature method, which are presented in Section 4. A function which does not satisfy any of the criteria to ensure membership of $\mathcal{G}[\Omega]$ is found, and numerical results suggest that the conditions are indeed fairly sharp. We also find that the rate of convergence degrades when the function has poles near $\Omega$.

Remark: The question of approximating $f$ by its derivatives has the feel of a classical problem. This author has not found any record of it being previously posed nor proved.

\section{Convergence}

In this section we develop several conditions for ensuring that $f \in \mathcal{G}[\Omega]$. We begin with the simplest form, in which $f$ is entire. A graphical representation of the proofs of Theorem 2.1 and Corollary 2.2 is given in Figure 1.

Theorem 2.1 If $\Omega$ is bounded, $f$ is entire and $f \in \mathcal{P}[\Omega]$, then $f \in \mathcal{G}[\Omega]$.

Proof:

Let $B_{k}=p_{k}+\left\{z:|z|<\epsilon_{k}\right\}$ be a sequence of balls for which $f \in \mathcal{P}[\Omega, \boldsymbol{B}]$. Without loss of generality, we can assume that $\epsilon_{k}<\epsilon$, zero is not in the closure of $\cup B_{k}$ and $\left|p_{k}\right| \rightarrow \infty$. 

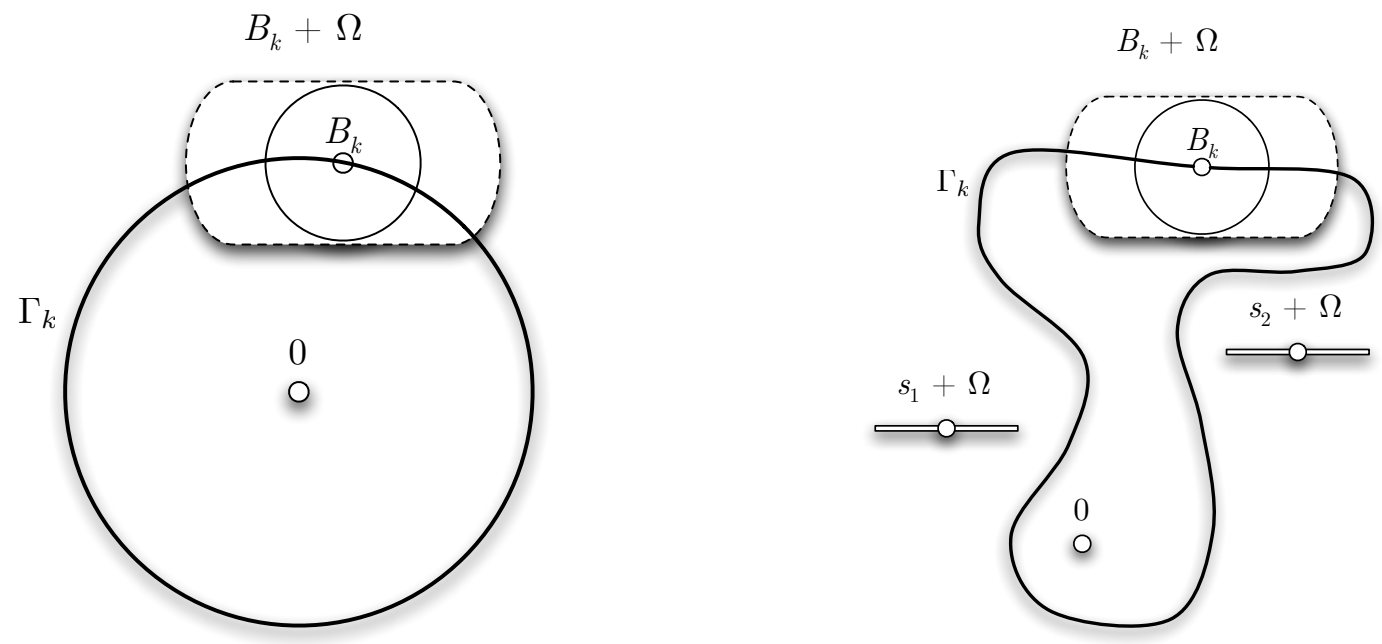

Figure 1: Diagrams to explain Theorem 2.1 (left) and Corollary 2.2 (right). In both of these diagrams, $\Omega$ is an interval centred around zero. On the right, $f$ has two singularities. The path $\Gamma_{k}$ goes around zero and through $B_{k}$, avoiding the singularities shifted by $\Omega$. This ensures that $\Gamma_{k}$ shifted by $\Omega$ avoids the singularities, and remains within $B_{k}+\Omega$ (the domain in dashed lines), the area in which $f$ decays.

Define the curve $\Gamma_{k}(t)=\left|p_{k}\right| \mathrm{e}^{\mathrm{i} t}$ for $0 \leq t \leq 2 \pi$. We will use the following smooth (but not analytic) bump function:

$$
\Lambda(z)= \begin{cases}\exp \left\{-\left(1-|z|^{2}\right)^{-2}\right\} & \text { if }|z|<1 \\ 0 & \text { otherwise }\end{cases}
$$

Define the function

$$
\tilde{1}_{k}(z)=1-\frac{2 \pi \mathrm{i}}{C_{k}} \Lambda\left(\frac{z-p_{k}}{\epsilon_{k}}\right)
$$

for

$$
C_{k}=\int_{\Gamma_{k} \cap B_{k}} z^{-1} \Lambda\left(\frac{z-p_{k}}{\epsilon_{k}}\right) d z=\frac{\epsilon_{k}}{p_{k}} \int_{\frac{\Gamma_{k} \cap B_{k}-p_{k}}{\epsilon_{k}}}\left(\frac{\epsilon_{k}}{p_{k}} u+1\right)^{-1} \Lambda(u) \mathrm{d} u .
$$

As $k \rightarrow \infty, \Gamma_{k} \cap B_{k}$ approaches a line segment of length $2 \epsilon_{k}$. Since $\Lambda$ is invariant under rotations, it follows that

$$
\frac{1}{C_{k}} \sim \frac{p_{k}}{\epsilon_{k}}\left[\int_{-1}^{1} \Lambda(u) \mathrm{d} u\right]^{-1}, \quad k \rightarrow \infty
$$

The function $\tilde{1}_{k}$ has the following important properties:

- It is identically one for all $z \notin B_{k}$; 
- The zeroth Laurent coefficient over $\Gamma_{k}$ is zero (note that what we refer to as a Laurent coefficient actually depends on $\Gamma_{k}$ since $\tilde{1}_{k}$ is not analytic):

$$
\oint_{\Gamma_{k}} \frac{\tilde{1}_{k}(z)}{z} d z=0
$$

- It is $C^{\infty}$ along $\Gamma_{k}$;

- We know a bound as $k$ increases (for some constant $P$ ):

$$
\left|\tilde{1}_{k}(z)\right| \leq 1+\frac{2 \pi}{\left|C_{k}\right|}\|\Lambda\|_{B_{k}} \sim 1+P \frac{\left|p_{k}\right|}{\epsilon_{k}}, \quad k \rightarrow \infty
$$

For any fixed $k$ we can choose $n_{k}$ large enough and constants $\left\{c_{-n_{k}}, \ldots, c_{n_{k}}\right\}$ so that

$$
\left\|\tilde{1}_{k}^{n_{k}}-\tilde{1}\right\|_{\Gamma_{k}} \leq\left[\left|\Gamma_{k}\right|\|f\|_{\Gamma_{k}+\Omega}\left\|\frac{1}{z}\right\|_{\Gamma_{k}} k\right]^{-1}
$$

where

$$
\tilde{1}_{k}^{n}=\sum_{j=-n}^{n} c_{j} z^{j}
$$

and $\left|\Gamma_{k}\right|=2 \pi\left|p_{k}\right|$ is the arclength of $\Gamma_{k}$. We can assume that $c_{0}$ is zero since the zeroth Laurent coefficient over $\Gamma_{k}$ of $\tilde{1}_{k}$ is zero, and we are only approximating $\tilde{1}_{k}$ on $\Gamma_{k}$.

For $x \in \Omega$,

$$
\begin{aligned}
2 \pi\left|f(x)-\sum_{j=1}^{n_{k}} \frac{c_{-j}}{j !} f^{(j)}(x)\right| & =\left|\oint_{\Gamma_{k}+x} \frac{f(z)}{z-x}\left(1-\sum_{j=-n_{k}}^{-1} c_{j}(z-x)^{j}\right) d z\right| \\
& =\left|\oint_{\Gamma_{k}} \frac{f(z+x)}{z}\left(1-\sum_{j=-n_{k}}^{-1} c_{j} z^{j}\right) d z\right|
\end{aligned}
$$


Due to analyticity, the integral $\oint_{\Gamma_{k}} \frac{f(z+x)}{z} z^{j} d z=0$ for $j=1,2, \ldots$, hence

$$
\begin{aligned}
(2.2) & =\left|\oint_{\Gamma_{k}} \frac{f(z+x)}{z}\left(1-\sum_{j=-n_{k}}^{n_{k}} c_{j} z^{j}\right) d z\right|=\left|\oint_{\Gamma_{k}} \frac{f(z+x)}{z}\left(1-\tilde{1}_{k}^{n_{k}}\right) d z\right| \\
& =\left|\oint_{\Gamma_{k}} \frac{f(z+x)}{z}\left(1-\tilde{1}_{k}\right) d z+\oint_{\Gamma_{k}} \frac{f(z+x)}{z}\left(\tilde{1}_{k}-\tilde{1}_{k}^{n_{k}}\right) d z\right| \\
& =\left|\oint_{\Gamma_{k} \cap B_{k}} \frac{f(z+x)}{z}\left(1-\tilde{1}_{k}\right) d z+\oint_{\Gamma_{k}} \frac{f(z+x)}{z}\left(\tilde{1}_{k}-\tilde{1}_{k}^{n_{k}}\right) d z\right| \\
& \leq\left|\Gamma_{k} \cap B_{k}\right|\|f\|_{\Gamma_{k} \cap B_{k}+\Omega}\left\|\frac{1-\tilde{1}_{k}}{z}\right\|_{\Gamma_{k} \cap B_{k}}+\left|\Gamma_{k}\right|\|f\|_{\Gamma_{k}+\Omega}\left\|\frac{1}{z}\right\|\left\|_{\Gamma_{k}}\right\| \tilde{1}_{k}-\tilde{1}_{k}^{n_{k}} \|_{\Gamma_{k}} \\
& \leq 2 \pi \epsilon_{k}\|f\|_{B_{k}+\Omega}\left[\left\|\frac{1}{z}\right\|_{B_{k}}+\left\|\frac{\tilde{1}_{k}}{z}\right\|_{B_{k}}\right]+k^{-1} .
\end{aligned}
$$

Since $f \in \mathcal{P}[\Omega, \boldsymbol{B}]$, we know that $\|f\|_{\Omega+B_{k}}=o(1)$. Combining (2.1) and the fact that $\left\|\frac{1}{z}\right\|_{B_{k}} \leq\left(\left|p_{k}\right|-\epsilon_{k}\right)^{-1}$ we obtain

$$
\epsilon_{k}\left\|\frac{\tilde{1}_{k}}{z}\right\|_{B_{k}} \leq \epsilon_{k}\left\|\tilde{1}_{k}\right\|_{B_{k}}\left\|\frac{1}{z}\right\|_{B_{k}}=\mathcal{O}\left(\frac{P\left|p_{k}\right|+\epsilon_{k}}{\left|p_{k}\right|-\epsilon_{k}}\right)=\mathcal{O}(1)
$$

We thus obtain

$$
\begin{aligned}
\left\|f-\sum_{j=1}^{n_{k}} \frac{c_{-j}}{j !} f^{(j)}\right\|_{\Omega} & \leq \epsilon_{k}\|f\|_{B_{k}+\Omega}\left[\left\|\frac{1}{z}\right\|_{B_{k}}+\left\|\frac{\tilde{1}_{k}}{z}\right\|_{B_{k}}\right]+(2 \pi k)^{-1} \\
& =o(1) \mathcal{O}(1)+\mathcal{O}\left(k^{-1}\right)=o(1) .
\end{aligned}
$$

Thus, by choosing $k$ large enough, we can approximate $f$ by its derivatives to any desired accuracy.

Q.E.D.

If $f$ is not entire, we must ensure that the curve $\Gamma_{k}$ shifted by each point in $\Omega$ remains within the domain of analyticity. The ability to do this depends on conditions on the domain of analyticity of $f$. In the following theorem, we allow $\Omega$ to be unbounded. If $\Omega$ is bounded, then $f$ is automatically bounded in $u+\Omega$ for every $u \in U$.

Corollary 2.2 Suppose $f$ is analytic in an open connected domain $D$ and that $f \in \mathcal{P}[\Omega, \boldsymbol{B}]$, where $B_{k}$ and the point zero lie within an open connected domain $U$ such that $U+\Omega \subset D$. If $\|f\|_{u+\Omega}<\infty$ for every $u \in U$, then $f \in \mathcal{G}[\Omega]$. 
Proof:

Let $S=\mathcal{C} \backslash D$. We must deform the curves $\Gamma_{k}$ to avoid the domain $S+\Omega$, so that shifting $\Gamma_{k}$ by any point in $\Omega$ remains within $D$. This is accomplished by choosing a smooth Jordan curve $\Gamma_{k}$ so that it remains within $U$, but still encloses zero and passes through $p_{k}$. We know such a curve exists since $U$ is open and connected, and the hypotheses ensure that $\Gamma_{k}+\Omega \subset D$.

Without loss of generality, we can assume that $\left|\Gamma_{k} \cap p_{k}+B\right|$ is still bounded by $2 \pi \epsilon_{k}$ and that $\Gamma_{k} \cap p_{k}+B$ approaches a straight line segment of length $2 \epsilon_{k}$ which passes through $p_{k}$ as $k \rightarrow \infty$. The hypotheses also ensure that $\|f\|_{\Gamma_{k}+\Omega}<\infty$. The proof is now equivalent to the proof of Theorem 2.1, with the following minor difference: $\left|\Gamma_{k}\right|$ is not necessarily equal to $2 \pi\left|p_{k}\right|$.

Q.E.D.

In applications the domain $\Omega$ is often an interval, in which case we can simplify the hypotheses of Corollary 2.2:

Corollary 2.3 If $\Omega$ is an interval, $f$ is analytic everywhere in the complex plane except at a finite set of points $S$ and $f \in \mathcal{P}[\Omega]$, then $f \in \mathcal{G}[\Omega]$.

Proof: $\quad$ We can assume that $B_{k} \cap S+\Omega=\varnothing$ for all $k$. A geometric argument states that it is impossible for $S+\Omega$ to cut off zero from $B_{k}$. Thus this corollary follows from Corollary 2.2.

Q.E.D.

An alternative situation in which one can guarantee convergence was suggested and proved to the author by Thomas Ransford [8]. We provide it here for reference, though we will use the versions based on $\mathcal{P}[\Omega]$ in our examples.

Theorem $2.4[8]$ Suppose that $\Omega$ is an interval and $f$ is entire and has only polynomial growth on $\mathbb{R} . f \in \mathcal{G}[\Omega]$ if and only if $f$ is not of the form

$$
f(x)=\sum_{j=0}^{n} a_{j} x^{j}+\sum_{j=0}^{n} \sum_{k=1}^{m} b_{j k} x^{j} \mathrm{e}^{\lambda_{k} x},
$$

where $a_{n} \neq 0$ and $\lambda_{k} \neq 0$ and imaginary for all $k$.

\section{Fast convergence for oscillatory integrals}

The preceding section demonstrates that for many functions differential GMRES will converge to the exact solution. In this section we explore the use of this new method for 
computing integrals for which traditional methods have difficulty. For reasons we shall explain, differential GMRES is extraordinarily efficient for oscillatory integrals of the form

$$
f(x)=r(x) \mathrm{e}^{\mathrm{i} \omega g(x)},
$$

where $r$ and $g$ are nonoscillatory. As long as turning points are avoided, WKBJ analysis ensures that many differential equations have solutions of this form, for example the Hankel function solutions of the Bessel equation [1].

Many advances have been made recently for the computation of such integrals; for example Filon-type methods [4], moment-free Filon-type methods [7], Levin-type methods [6] and numerical steepest descent $[\mathbf{3}]$. Unfortunately, each of these methods requires knowledge of the oscillator $g$, whereas differential GMRES only requires knowledge of the function $f$ (and its derivatives) at a sequence of points $\left\{x_{1}, \ldots, x_{m}\right\}$.

There has been an issue that has been avoided so far: the $L_{2}$ inner product itself requires the computation of integrals. Instead, we use the following discrete inner product when $\Omega$ is an interval:

$$
\langle f, g\rangle=\sum_{i=1}^{m} w_{i} f\left(x_{i}\right) \bar{g}\left(x_{i}\right)
$$

where $\left\{x_{1}, \ldots, x_{m}\right\}$ and $\left\{w_{1}, \ldots, w_{m}\right\}$ are Gauss quadrature nodes and weights, respectively. We will initially use Gauss-Lobatto quadrature. At first glance, one might assume that it would be better to use this quadrature formula directly. But we will see that differential GMRES can significantly outperform such a method. We denote the differential GMRES approximation using an $m$ point Gauss-Lobatto formula to compute the inner products by $v_{n, m}^{\mathrm{G}}$, and its use as a quadrature formula over $(a, b)$ as

$$
Q_{n, m}^{\mathrm{G}}=v_{n, m}^{\mathrm{G}}(b)-v_{n, m}^{\mathrm{G}}(a)
$$

We continue to use the notation $v_{n}^{\mathrm{G}}$ and $Q_{n}^{\mathrm{G}}$ for when the exact $L_{2}$ inner product is used.

The key to the success of differential GMREs is that the derivatives of $f$ automatically capture the oscillations of $f$ :

$$
f^{\prime}=\left(r^{\prime}+\mathrm{i} g^{\prime} r\right) \exp (\mathrm{i} \omega g), \quad f^{\prime \prime}=\left(r^{\prime \prime}+\mathrm{i} g^{\prime \prime} r+2 \mathrm{i} g^{\prime} r^{\prime}-g^{\prime 2} r\right) \exp (\mathrm{i} \omega g), \quad \ldots
$$

Thus we can write $q_{1}, q_{2} \in \mathcal{K}_{n}[f]$ as $r_{1} \exp (\mathrm{i} \omega g)$ and $r_{2} \exp (\mathrm{i} \omega g)$, where $r_{1}$ and $r_{2}$ are nonoscillatory. It follows that the inner product of these two functions is a nonoscillatory integral

$$
\left\langle q_{1}, q_{2}\right\rangle=\int r_{1} \bar{r}_{2} \mathrm{e}^{\mathrm{i} \omega g} \mathrm{e}^{-\mathrm{i} \omega g} \mathrm{~d} x=\left\langle r_{1}, r_{2}\right\rangle
$$

Since the inner products used in GMRES are always between members of $\mathcal{K}_{n}[f],(3.1)$ requires only a small number of points to achieve high accuracy. 

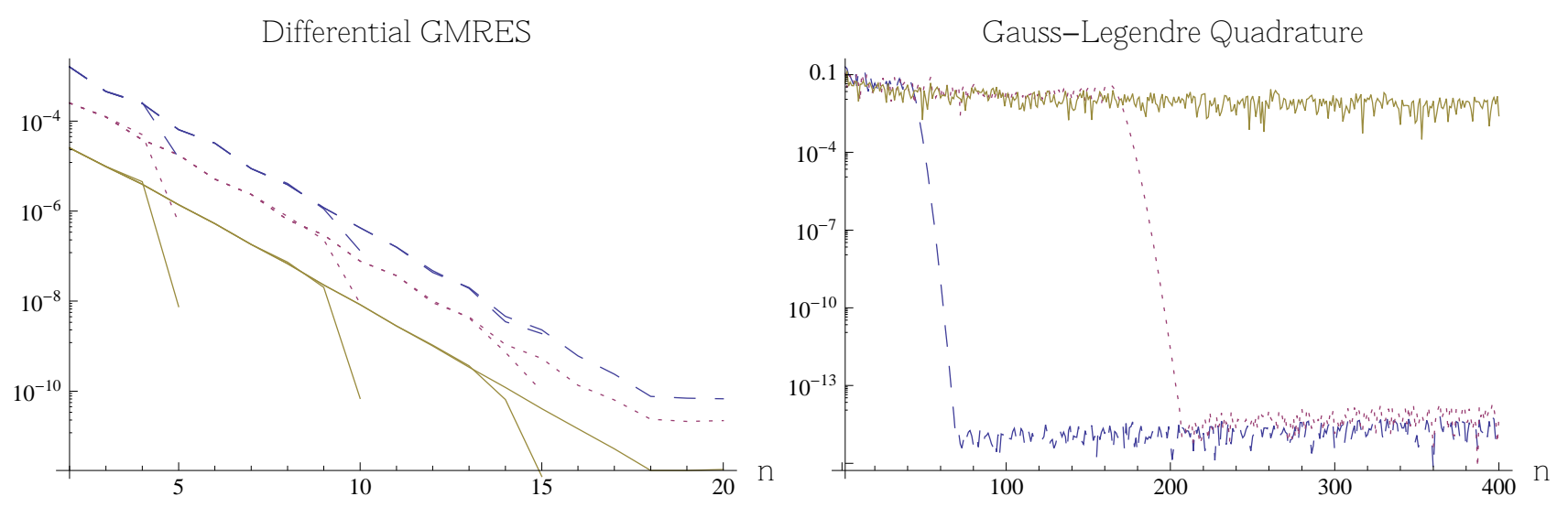

Figure 2: The errors in approximating $\int_{1}^{2}\left[\mathrm{Ai}\left(-\omega x^{2}\right)+\mathrm{iBi}\left(-\omega x^{2}\right)\right] \mathrm{d} x$ by $Q_{n, m}^{\mathrm{G}}$ for $m=$ 5, 10,15, 20 (left) and an $n$-point Gauss-Legendre rule (right), for $\omega=10$ (dashed), 25 (dotted), and 100 (solid). Gauss-Legendre quadrature stagnates for large $\omega$, whilst differential GMRES becomes more accurate.

As an example, consider the integral

$$
\int_{1}^{2}\left[\mathrm{Ai}\left(-\omega x^{2}\right)+\mathrm{iBi}\left(-\omega x^{2}\right)\right] \mathrm{d} x
$$

Both $\mathrm{Ai}$ and Bi decay along the negative real axis and therefore convergence over an interval is assured by Theorem 2.1. When $\omega$ is large, the oscillations of the Airy functions are approximately trigonometric $[\mathbf{1}]$ :

$$
\begin{aligned}
& \mathrm{Ai}(-\omega x) \sim \frac{\sin \left(2 / 3(-\omega x)^{3 / 2}+\pi / 4\right)}{\sqrt{\pi}(-\omega x)^{1 / 4}}, \\
& \operatorname{Bi}(-\omega x) \sim \frac{\cos \left(2 / 3(-\omega x)^{3 / 2}+\pi / 4\right)}{\sqrt{\pi}(-\omega x)^{1 / 4}} .
\end{aligned}
$$

It follows that $\mathrm{Ai}\left(-\omega x^{2}\right)+\mathrm{iBi}\left(-\omega x^{2}\right)$ is asymptotic to an exponential oscillator. For this function requiring knowledge of derivatives is not restrictive: An Airy function satisfies a straightforward differential equation, and its derivatives can be written in terms of itself, its first derivative and polynomials.

Figure 2 compares the error of $Q_{n, m}^{\mathrm{G}}$ for three choices of $\omega$ and four choices of $m$ to that of Gauss-Legendre quadrature, where the exact solution is computed to machine precision using MATHEMATICA's NIntegrate routine. Note how $Q_{n, m}^{\mathrm{G}}$ actually improves with accuracy as $\omega$ increases, where Gauss-Legendre quadrature stagnates. $Q_{n, m}^{\mathrm{G}}$ uses $m n$ pieces of data and thus a proper comparison is between last data point in the first graph with the point $n=400$ in the second graph. The reason there is a jump in accuracy when $n=m$ is that when we interpolate at the endpoints of an oscillatory integral the asymptotic order increases by one. This follows from a single integration by parts, as explained in [4]. This motivated our use of 

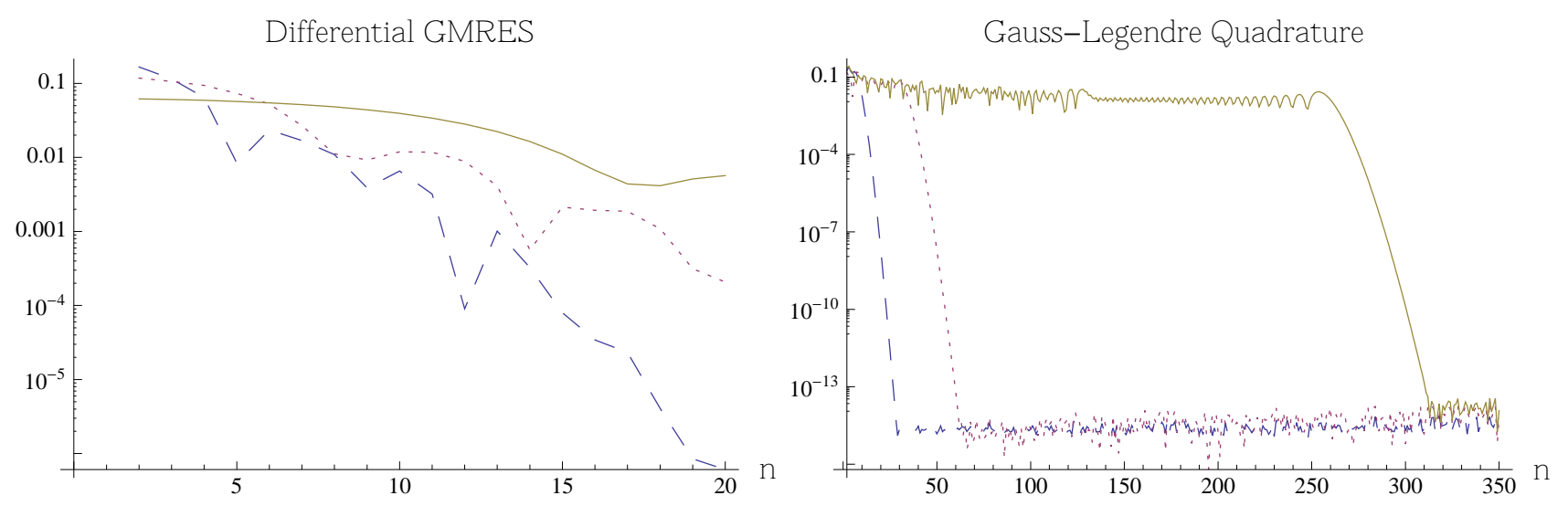

Figure 3: The errors in approximating $\int_{0}^{1}\left[\mathrm{Ai}\left(-\omega x^{2}\right)+\mathrm{iBi}\left(-\omega x^{2}\right)\right] \mathrm{d} x$ by $Q_{n}^{\mathrm{G}}$ (left) and an $n$-point Gauss-Legendre rule (right), for $\omega=10$ (dashed), 25 (dotted) and 100 (solid).
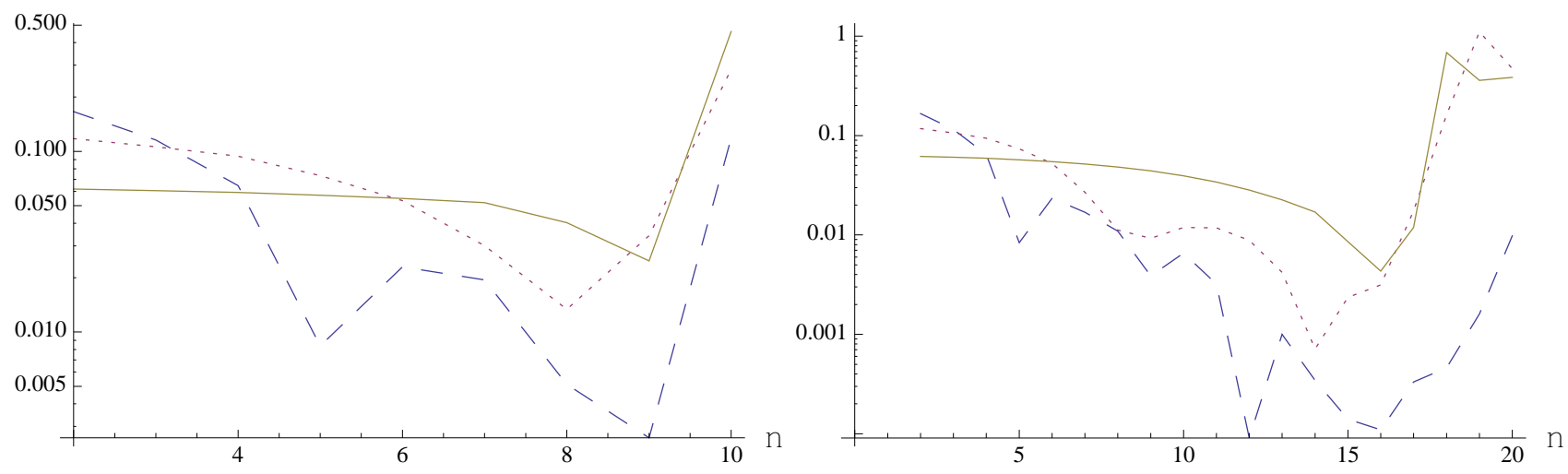

Figure 4: The errors in approximating $\int_{0}^{1}\left[\mathrm{Ai}\left(-\omega x^{2}\right)+\mathrm{iBi}\left(-\omega x^{2}\right)\right] \mathrm{d} x$ by $Q_{n, 10}^{\mathrm{G}}$ (left) and $Q_{n, 20}^{\mathrm{G}}$ (right) for $\omega=10$ (dashed), 25 (dotted) and 100 (solid).

Gauss-Lobatto quadrature; otherwise we would not interpolate at the endpoints and not see an increase in accuracy. For differential GMRES the relative error is bounded when $n \neq m$ and decays when $n=m$, while it grows for Gauss-Legendre quadrature.

Remark: These results suggest the following seemingly sensible scheme: fix $m$, compute $v_{m, m}^{\mathrm{G}}$ and then restart the GMRES algorithm on the residual $f-\mathcal{D} v_{m, m}^{\mathrm{G}}$ now using, say, $2 m$ points for the inner products. Unfortunately, in the numerical tests so far conducted the error often stagnates after restarting.

In the preceding example, we intentionally avoided the turning point at $x=0$. The reason for this is demonstrated in the disappointing results shown in Figure 3 and Figure 4. Now as $\omega$ increases the rate of convergence slows down. Worse, as the GMRES iteration count approaches the number of sample points, the error drastically grows. Before, the fact that the function was (very close to) an exponential oscillator meant that the inner products were 


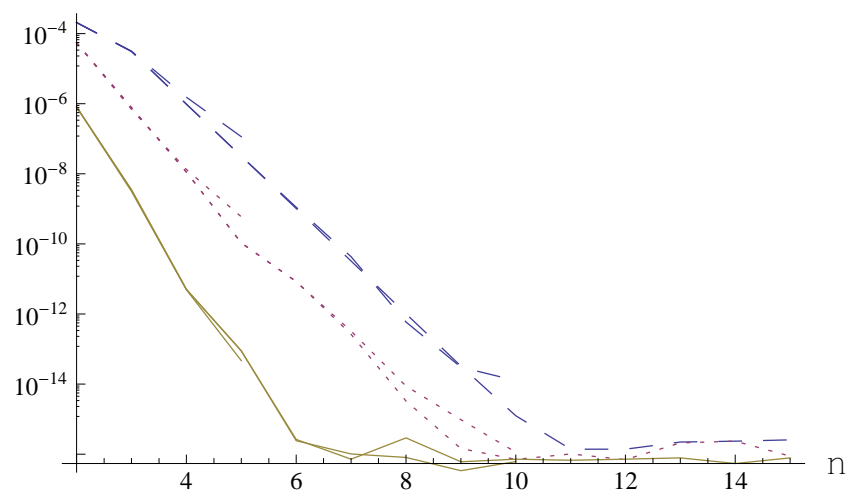

Figure 5: The error in approximating $\int_{0}^{1} \frac{x+1}{x-2} \mathrm{e}^{\mathrm{i} \omega x} \mathrm{~d} x$ by $Q_{n, m}^{\mathrm{G}}$ for $m=5,10,15$ and $\omega=10$ (dashed), 25 (dotted), and 100 (solid).

nonoscillatory. This is no longer the case, and thus a sufficient number of sample points must be used to resolve the oscillations. The accuracy is not much improved when the discrete inner products are replaced by $L_{2}$ inner products, though the error no longer blows up as the iteration count increases.

Perhaps the most important special case of an exponential oscillator is a Fourier oscillator, i.e., when $g(x)=x$. Figure 5 utilizes differential GMREs to approximate the integral

$$
\int_{0}^{1} \frac{x+1}{x-2} \mathrm{e}^{\mathrm{i} \omega x} \mathrm{~d} x
$$

Remarkably, not only does the accuracy increase as $\omega$ grows, but in fact the error decays like $\mathcal{O}\left(\omega^{-n-1}\right), \omega \rightarrow \infty$. This has a straightforward explanation: an asymptotic expansion for a particular solution to the equation is

$$
\left\|f-\mathcal{D} v_{n}^{A}\right\|_{\Omega}=\mathcal{O}\left(\omega^{-n}\right) \quad \text { for } \quad v_{n}^{A}(x)=-\sum_{k=1}^{n} \frac{f^{(k-1)}(x)}{(-\mathrm{i} \omega)^{k}} \mathrm{e}^{\mathrm{i} \omega x}
$$

since $v_{n}^{A} \in \mathcal{K}_{n}[f]$, it follows that

$$
\left\|f-\mathcal{D} v_{n}^{\mathrm{G}}\right\|_{\Omega} \leq\left\|f-\mathcal{D} v_{n}^{A}\right\|_{\Omega}=\mathcal{O}\left(\omega^{-n}\right)
$$

Indeed, by using an appropriate preconditioner, we can achieve the same asymptotic decay whenever the kernel is $\mathrm{e}^{\mathrm{i} \omega g(x)}$ for $g^{\prime}(x) \neq 0$ for all $x \in \Omega$. This will be the subject of a future paper.

Finally, consider the example

$$
E_{1}(\mathrm{i} \omega)=\int_{1}^{\infty} \frac{\mathrm{e}^{-\mathrm{i} \omega x}}{x} \mathrm{~d} x
$$

where $E_{1}$ is the Exponential integral [1]. Again the kernel of oscillations is a Fourier oscillator, but now we are integrating over an infinite region. We can no longer use Gauss-Lobatto 


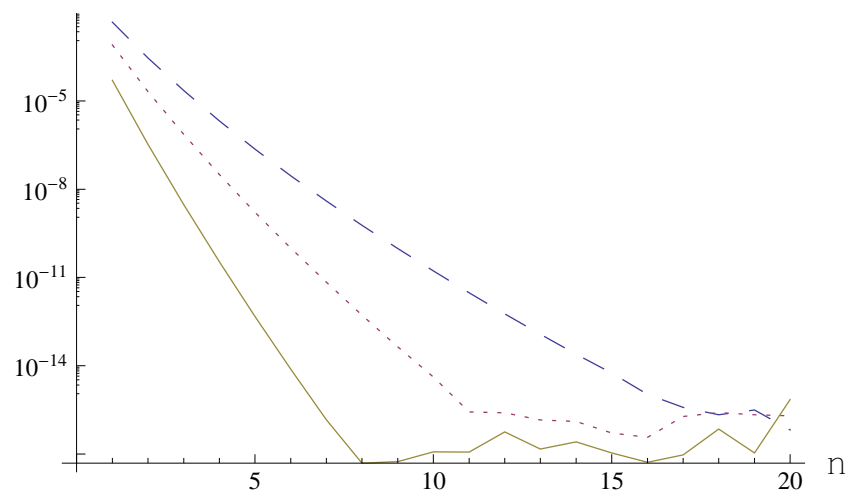

Figure 6: The error in approximating $E_{1}(\mathrm{i} \omega)$ by $Q_{n}^{\mathrm{G}}$ for $\omega=10$ (dashed),25 (dotted) and 100 (solid).

quadrature for computing the inner products, and Gauss-Laguerre quadrature does not perform well in practice. Thus instead we use the continuous $L_{2}$ inner product. Figure 6 shows that differential GMRES still works well, even with an unbounded domain.

\section{Counterexamples}

In this section we present some functions which do not satisfy any of the criteria that ensure membership of $\mathcal{G}[\Omega]$, to give an idea of the limits of differential GMRES. We also find functions where the convergence rate of differential GMRES slows down drastically. We have already mentioned the most important example: $p$ is not in $\mathcal{G}[\Omega]$ if $p$ is a polynomial. As a result, if we expand a general function $f$ into a Chebyshev series $p=\sum_{0}^{n} c_{k} T_{k}$-say, using the chebfun framework in MATLAB [2] - and attempt to apply differential GMRES, the method will not converge. There are three factors which make this issue less significant:

- We already know an antiderivative of a polynomial in closed form, so by expanding into a polynomial we automatically obtain a solution to (1.1);

- For Chebyshev series the remaining error is at most $\left|c_{n}\right|$, which should typically be small;

- If we are solving any other first order differential equation, then the conditions are often satisfied since $x^{k} \mathrm{e}^{a(x)}$ typically decays in the complex plane.

Corollary 2.2 states that we must be able to reach the region in which $f$ decays in an open domain $U$ such that $U+\Omega$ avoids all the singularities of $f$. Consider the following example:

$$
f(z)=[-\exp (-2 \pi \mathrm{i} z)+\exp (2 \pi)]^{-1} .
$$

This decays for large positive imaginary $z$, but it has poles at $S=\mathbb{Z}+\mathrm{i}$. Thus if $\Omega$ is an interval of width greater than one $S+\Omega$ is the line $\mathbb{R}+\mathrm{i}$, and it is impossible to construct 


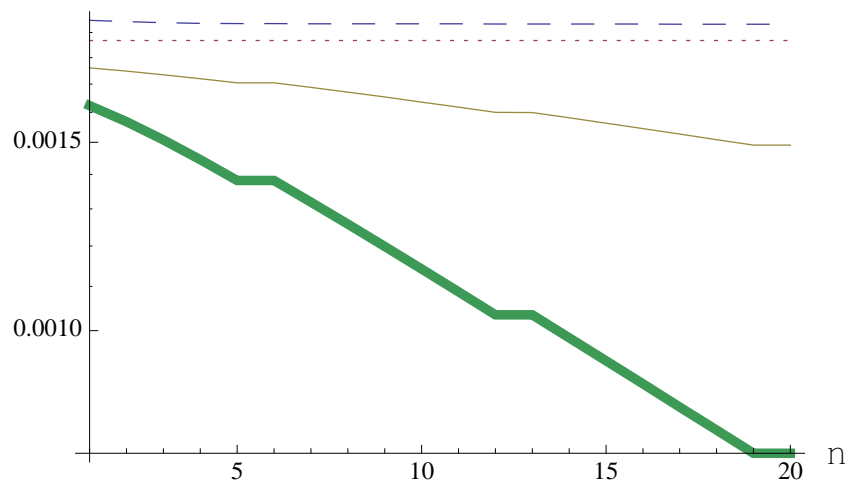

Figure 7: The $L_{2}$ error in approximating $[-\exp (-2 \pi \mathrm{i} z)+\exp (2 \pi)]^{-1}$ by $v_{n}^{\mathrm{G}}$ over $[0,1.1]$ (dashed), [0,1] (dotted), [0, 0.9] (solid) and [0,0.8] (thick).
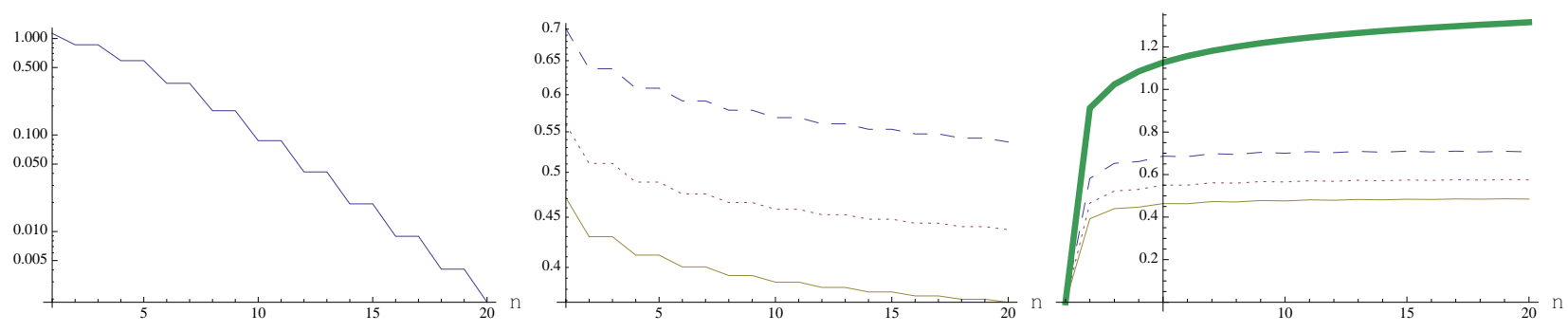

Figure 8: In the first two graphs, the $L_{2}$ error in approximating $\frac{1}{q x^{2}+1}$ by $v_{n}^{\mathrm{G}}$ over $[-1,1]$ for $q=1$ (left), 10 (dashed), 25 (dotted) and 50 (solid). In the right graph we scale the errors of the middle graph by $(\log n)^{1 / 4}$. We also plot $(\log n)^{1 / 4}$ (thick) for comparison.

such a set $U$. If, on the other hand, $\Omega$ is an interval of size less than one then Corollary 2.2 applies and $f \in \mathcal{G}[\Omega]$. The norm of the residual $\left\|f-\mathcal{D} v_{n}^{\mathrm{G}}\right\|$ for four choices of intervals is depicted in Figure 7.

Another difficult situation is

$$
f(z)=\frac{1}{q z^{2}+1}, \quad \Omega=[-1,1] .
$$

When $q$ is large this function has poles near the interval. In this case differential GMRES does converge, but the poles near $\Omega$ cause the rate of convergence to decrease drastically. Figure 8 shows how the method breaks down once $q$ passes a certain threshold. When $q=1$ it appears that differential GMRES achieves spectral convergence, whereas the errors for the three values tested with $q \geq 10$ appear to decay roughly like $\mathcal{O}\left((\log n)^{-1 / 4}\right)$.

One final example over an unbounded domain is

$$
f(z)=\frac{\mathrm{e}^{\mathrm{i} z^{2}}}{z}, \quad \Omega=[1, \infty)
$$


The method cannot possibly converge as the higher order derivatives of $f$ are unbounded in $\Omega$. On the other hand, we are very close to satisfying the criteria, since $f$ decays in the first quadrant of the complex plane and is bounded in $\Omega$. The problem is that $f$ grows exponentially for any small perturbation into the fourth quadrant (positive real value and negative imaginary value). For any open set $U$ containing $\Omega$, we can choose $u \in U$ such that $\operatorname{Im} u<0$, in which case $f$ is unbounded in $u+\Omega$.

\section{Closing Remarks}

We have seen that in many cases differential GMREs will converge and can be used successfully to integrate oscillatory functions, even when the kernel of oscillations is unknown. When the kernel is a Fourier oscillator, the method converges spectacularly fast. A future paper will introduce a preconditioner to obtain this fast convergence for other oscillators. A second future paper will investigate using this method to solve certain higher order oscillatory differential equations.

Differential GMRES depends on derivatives, which we have computed analytically using MathematicA. Often in applications the integrand is a solution to a differential equation, in which case this requirement is not prohibitive. Otherwise, we can approximate the function $f$ by another function $p$ such that the derivatives of $p$ are computable, and apply differential GMRES to $p$. The fact that the derivatives of $p$ are not necessarily accurate approximations to the derivatives of $f$ is immaterial. If $f$ has the form $r(x) \mathrm{e}^{\mathrm{i} \omega g(x)}$ we can approximate the nonoscillatory component $r$ by a function $v$ and take $p=v \mathrm{e}^{\mathrm{i} \omega g}$. Theorem 2.1 suggests that the convergence rate depends somehow on a functions decay in the complex plane, so it is probably more accurate to let $v$ be, say, a rational function instead of a polynomial.

There are several questions we have left unanswered. Can other Krylov subspace methods (such as BCGSTAB) be used? What determines the convergence rate? Can the use of a preconditioner increase the convergence rate of oscillatory integrals with turning points? Can the method be generalized to solve vector-valued differential equations of the form

$$
\boldsymbol{v}^{\prime}(t)=A(t) \boldsymbol{v}(t)
$$

where $A(t)$ has large imaginary eigenvalues? Can we use it to integrate over multidimensional domains? It is our feeling that this method has great potential, especially if these questions can be answered satisfactorily.

Acknowledgments: The author thanks Nick Trefethen, who gave valuable advice on the content and direction of this paper. He also thanks Charles Batty, Toby Driscoll, Arieh Iserles and Thomas Ransford. 


\section{References}

[1] Abramowitz, M. and Stegun, I., Handbook of Mathematical Functions, National Bureau of Standards Appl. Math. Series, \#55, U.S. Govt. Printing Office, Washington, D.C., 1970.

[2] Battles, Z. and Trefethen, L.N., An extension of Matlab to continuous functions and operators, SIAM J. Sci. Comput. 25 (2004), 1743-1770.

[3] Huybrechs, D. and Vandewalle, S., On the evaluation of highly oscillatory integrals by analytic continuation, SIAM J. Num. Anal. 44 (2006), 1026-1048.

[4] Iserles, A. and Nørsett, S.P., Efficient quadrature of highly oscillatory integrals using derivatives, Proceedings Royal Soc. A 461 (2005), 1383-1399.

[5] Nevanlinna, O., Convergence of Iterations for Linear Equations, Birkhäuser, Basel, Boston, 1993.

[6] Olver, S., Moment-free numerical integration of highly oscillatory functions, IMA J. Num. Anal. 26 (2006), 213-227.

[7] Olver, S., Moment-free numerical approximation of highly oscillatory integrals with stationary points, Euro. J. Appl. Math. 18 (2007), 435-447.

[8] Ransford, T., personal communication, 2008.

[9] Trefethen, L.N. and Bau III, D., Numerical Linear Algebra, SIAM, 1997, 191-192. 